\title{
Dispersal of Amblyseius swirskii Athias-Henriot (Acari: Phytoseiidae) on potted greenhouse chrysanthemum
}

\author{
Rosemarije Buitenhuis $^{\mathrm{a}, \mathrm{b}}$, Les Shipp ${ }^{\mathrm{b}, *}$, Cynthia Scott-Dupree ${ }^{\mathrm{a}}$ \\ a Department of Environmental Biology, University of Guelph, Guelph, Ont., Canada N1G 2W1 \\ ${ }^{\mathrm{b}}$ Greenhouse and Processing Crops Research Centre, Agriculture and Agri-Food Canada, Harrow, Ont., Canada
}

\section{A R T I C L E I N F O}

Article history:

Received 24 March 2009

Accepted 18 October 2009

Available online 22 October 2009

\section{Keywords:}

Dispersal

Amblyseius swirskii

Frankliniella occidentalis

Potted chrysanthemum

Greenhouse

Biological control

\begin{abstract}
A B S T R A C T
Dispersal of natural enemies through a crop is a key component of biological control. The release strategy should optimize the number of predators that are released, the release frequency and number of release sites throughout a crop with regards to the distance that natural enemies can disperse from their release point. In this study, dispersal rate and behaviour of Amblyseius swirskii Athias-Henriot (Acari: Phytoseiidae) was investigated in potted greenhouse chrysanthemums in the presence or absence of prey (Frankliniella occidentalis (Pergande) (Thysanoptera: Thripidae). Results demonstrate that A. swirskii did not disperse far from the release site. Presence of prey did not influence dispersal, but had an effect on predator survival in one experiment. Only a quarter of the A. swirskii eventually attempted to disperse by going down to the ground. The presence of inter-plant contact greatly improved movement of $A$. swirskii between plants. It is concluded that good coverage with predators of the crop is needed when using $A$. swirskii in a biological control program. Having a continuous crop canopy will promote dispersal.
\end{abstract}

Crown Copyright $\odot 2009$ Published by Elsevier Inc. All rights reserved.

\section{Introduction}

Dispersal of natural enemies throughout a crop is a key component of biological control. In many crops it is important to suppress pests to very low levels quickly, often using a preventative approach. Consequently, patches of prey will be sparsely distributed within a crop and successful biological control will rely on the speed with which the biological control agent can move into and between these isolated patches (Skirvin and Fenlon, 2003). In greenhouses, especially in ornamentals most biological control programs rely on regular augmentative releases of natural enemies. Repeated releases are necessary because there is often not enough prey to maintain a constant predator population (Van Driesche and Heinz, 2004). In order to obtain satisfactory and costeffective control, the release strategy should be optimized regarding the number of predators that are released, the release frequency and number of release sites throughout the greenhouse.

Mites of the family Phytoseiidae are used as biological control agents on various crops (McMurtry and Croft, 1997). Amblyseius swirskii (Athias-Henriot) (Acari: Phytoseiidae) is a generalist predator and is used for whitefly and thrips control in greenhouse crops (Messelink et al., 2006; Nomikou et al., 2001). Commercially pro-

\footnotetext{
* Corresponding author. Address: Greenhouse and Processing Crops Research Centre, Agriculture and Agri-Food Canada, 2585 County Rd. 20, Harrow, Ont., Canada NOR 1G0. Fax: +1 5197382929.

E-mail addresses: Les.Shipp@agr.gc.ca, shippl@agr.gc.ca (L. Shipp).
}

duced A. swirskii can be released by different methods, most of them involving release from several point sources. Slow-release sachets are small breeding units that are hung on the plant and produce predators over several weeks. Predatory mites in a bran or vermiculite carrier are generally placed as piles on the leaves or substrate. Alternatively, this loose product can be broadcast, e.g., by air blast (Opit et al., 2005). To obtain an even distribution of predators throughout the crop, the release strategy should take into account the distance that the predators move from the release site. In general, predatory mites move between plants by ambulatory and aerial means (Croft and Jung, 2001). In outdoor crops, the predatory mites can disperse quickly through a crop due to aerial dispersal (Jung and Croft, 2001). However, in greenhouses the primary means of dispersal for predatory mites is by ambulatory movement because wind speed in the greenhouse is often too low for effective aerial dispersal (Zemek and Nachman, 1999). Skirvin and Fenlon (2003) found that for Phytoseiulus persimilis AthiasHenriot (Acari: Phytoseiidae) the risk of mortality during dispersal depends on inter-plant contact and type of ground substrate. When $P$. persimilis move between isolated plants, mortality risks are high and dispersal is restricted. However, when the plant canopy closes or inter-plant bridges are present, more $P$. persimilis move from plant to plant (Casey and Parrella, 2005). Another factor that influences dispersal is presence of prey. Several studies have shown that the dispersal rate of phytoseiid mites deceases with an increase in prey densities (Croft et al., 1995; Zemek and Nachman, 1998). 
The objective of this study was to measure movement of $A$. swirskii within greenhouses containing potted chrysanthemums. The following questions were investigated: In the presence or absence of prey, how far do A. swirskii disperse from a release plant and what routes do they take to get to the next plant? In addition, the influence of inter-plant contact on dispersal behaviour was investigated.

\section{Materials and methods}

\subsection{Plants, predatory mites and prey}

The plants used in the experiments were potted chrysanthemums, Dendranthema grandiflora (Tzvelev) var. Chesapeake, with yellow daisy-type flowers that were obtained from Yoder Canada (Leamington, Ontario, Canada). All chrysanthemums were grown in 15-cm-diameter pots with four to five cuttings per pot. A colony of Frankliniella occidentalis (Thysanoptera: Thripidae) was maintained in a greenhouse on flowering potted chrysanthemum (var. Chesapeake) to serve as prey in the experiments. To collect adult $F$. occidentalis for the trials, the plants were tapped above a white tray and adults and/or larvae (mixed ages) were aspirated into vials. A. swirskii was obtained from Biobest Canada Ltd. (Leamington, Ontario, Canada) as bulk product in a bran carrier and used immediately on arrival.

\subsection{Dispersal from a central release point with or without prey}

The experiment was conducted at Agriculture and Agri-Food Canada, Greenhouse and Processing Crops Research Centre (GPCRC) (Harrow, Ontario, Canada) from January to April 2008. Treatments were presence or absence of prey. Sampling was destructive, therefore separate experimental plots were set up for sampling on day 2, 5, 9 and 14. Experimental plots consisted of 37 plants that were set up in three circles $(6,12$ and 18 plants) around a central release plant. All plants were spaced approximately $30 \mathrm{~cm}$ apart, measured from the middle of the pots, which simulates commercial growing practices. At the beginning of the experiment, plants were vegetative in replicate 1 and 2 , and had small buds in replicate 3 and 4 . The foliage of neighbouring plants was slightly touching. Six identical greenhouses $(13 \times 7.5 \mathrm{~m})$ were used. Two replicates were done at the same time so each greenhouse contained two or three plots. To minimize movement of mites between plots in the same greenhouse, plots were separated by $1 \mathrm{~m}$ high plastic barriers that were taped to the ground and a layer of insect glue (Tangle-trap ${ }^{\circledR}$, The Tanglefoot Company, Grand Rapids, Michigan, USA) was applied to the tape. The treatments with prey were grouped together in three greenhouses to prevent infestation of the treatments without prey and sampling dates were randomly assigned to plots within greenhouses. The experiment was repeated once over time, so in total there were four replicates. Temperatures in the greenhouses during the study averaged $22^{\circ} \mathrm{C}$ with a range of $18-30{ }^{\circ} \mathrm{C}$.

One week before $A$. swirskii release, 20 adult thrips (18 females and 2 males) and 5 thrips larvae were released on each plant in the treatments with prey to establish a prey population in the plots. On the day of $A$. swirskii release, samples $(3 \times 1 \mathrm{ml})$ were taken from the $A$. swirskii bulk product; the number of $A$. swirskii per sample was determined and used to estimate the density of predators per $\mathrm{ml}$ in the product. Based on this estimate, small $(5 \mathrm{~cm}$ diameter) Petri dishes were prepared with approximately $250 \mathrm{~A}$. swirskii each. One Petri dish with predators was placed in the middle of each release plant. Four extra Petri dishes were prepared and sampled $(3 \times 1 \mathrm{ml})$ to estimate more precisely the actual number of predators that was released. In replication 1 and 2 , an average of
$260 \pm 19$ A. swirskii was released, in replication 3 and 4 this was $279 \pm 36$ A. swirskii.

On day 2, 5, 9 and 14 after $A$. swirskii release, all potted plants in plots were cut at the base of the stem and placed individually in labelled plastic bags. Samples were washed in 0.1\% Triton XL80N, filtered using a Büchner funnel, and number of $A$. swirskii and $F$. occidentalis (larvae and adults) were recorded per plant. All predatory mites were slide mounted in Hoyer's medium to confirm species identification. When plots were sampled, the Petri dishes from which the predators were released were recovered and the number of $A$. swirskii remaining in the dishes was estimated from the counts of three $1 \mathrm{ml}$ samples.

The data were used to calculate the mean distance traversed from the central release point (Jung and Croft, 2001).

\subsection{Dispersal routes with and without prey}

This experiment was designed to determine where the predators go after release on a plant. The experiment was conducted at the GPCRC from April to May 2008 in a $7.5 \times 7 \mathrm{~m}$ greenhouse. The experimental unit consisted of a non-flowering potted chrysanthemum plant (var. Chesapeake) in a cage. Treatments were the presence or absence of prey. One week before A. swirskii release, 20 adult thrips ( 18 females and 2 males) and 5 thrips larvae were released on each plant in the treatment with prey to establish a prey population. On the day of $A$. swirskii release, yellow sticky cards $(40 \times 50 \mathrm{~cm})$ were placed under the pots in the cages. As in the previous experiment, small $(5 \mathrm{~cm}$ diameter) Petri dishes were prepared with approximately $250 \mathrm{~A}$. swirskii each. One Petri dish with predators was placed in the middle of each release plant. Based on counts of extra Petri dishes with predators it was estimated that $251 \pm 21$ A. swirskii were released. Temperatures in the greenhouse during the study averaged $22{ }^{\circ} \mathrm{C}$ with a range of $18-32{ }^{\circ} \mathrm{C}$.

On day 2, 5, 9 and 14 after $A$. swirskii release, the plant was cut at the base of the stem and placed in a labelled plastic bag. Samples were washed and filtered as described in the previous experiment and number of $A$. swirskii and $F$. occidentalis (larvae and adults) were recorded per plant. The Petri dishes from which the predators were released were recovered and number of $A$. swirskii remaining in the dishes was estimated from the counts of three $1 \mathrm{ml}$ samples. The sticky cards under the pots were wrapped in clear plastic foil, examined under the microscope and number of $A$. swirskii recorded. The outside of the pot was rinsed with $70 \%$ alcohol which was then filtered using a Büchner funnel and numbers of $A$. swirskii were counted. Finally, the top $1 \mathrm{~cm}$ of soil was removed from the pot and placed on Berlese funnels to extract $A$. swirskii from the soil. The A. swirskii were caught in dishes containing water with $0.1 \%$ Triton XL80N that were placed under the Berlese funnels. This solution was filtered using a Büchner funnel and number of $A$. swirskii counted. The experiment was replicated three times.

\subsection{Effect of inter-plant contact on dispersal}

The aim of this experiment was to determine whether A. swirskii was more likely to disperse between plants when the plant canopies were touching (continuous canopy) or when it had to move down the pot, walk on the ground and move up the next pot. The experiment was conducted at the GPCRC in July 2008 in a $7.5 \times 15 \mathrm{~m}$ greenhouse. Two non-flowering potted chrysanthemums (var. Chesapeake) (a release and a receiving plant) were placed in a $60 \times 90 \mathrm{~cm}$ rectangle that was surrounded by tape coated with insect glue to prevent $A$. swirskii from leaving the experimental area. The plant canopies were either touching or non-touching $(10 \mathrm{~cm}$ apart). As in the previous experiments, small (5 $\mathrm{cm}$ diameter) Petri dishes were prepared with approximately 
250 A. swirskii each. One Petri dish with predators was placed in the middle of each release plant. Based on counts of extra Petri dishes with predators it was estimated that $154 \pm 15$ A. swirskii were released. Temperatures in the greenhouse during the study averaged $26{ }^{\circ} \mathrm{C}$ with a range of $18-34{ }^{\circ} \mathrm{C}$.

On day 2, 5, 9 and 14 after $A$. swirskii release, all potted plants in plots were cut at the base of the stem and placed individually in labelled plastic bags. Samples were washed as described in the first experiment and numbers of $A$. swirskii were recorded for the release and receiving plants. The Petri dishes from which the predators were released were recovered and the number of $A$. swirskii remaining in the dishes was estimated from the counts of three $1 \mathrm{ml}$ samples. The experiment was replicated five times.

\subsection{Statistical analysis}

The analysis has no repeated measurements. Separate plots were set up for 2, 5, 9 and 14 days because sampling was destructive. All data were analysed with two-way ANOVA with day and treatments as main factors using SAS (version 9.1.3) and differences were determined to be significant at $\alpha<0.05$. Where needed, data were transformed in order to normalize data and obtain homogeneity of variances.

\section{Results}

In all experiments, $>80 \%$ of the predators left the Petri dish with bran within 2 days. Recovery of $A$. swirskii ranged between $94 \%$ and $14 \%$ depending on the sampling date (2, 5, 9 or 14 days) and the experiment. Mean recovery per experiment was 56\%, 50\% and $31 \%$ for experiments 1,2 and 3 , respectively.

\subsection{Dispersal from a central release point with or without prey}

Only a small percentage of the released A. swirskii dispersed from the release plant to the neighbouring plants (Fig. 1). After 14 days, up to $15 \mathrm{~A}$. swirskii were found in the first circle around the release plant and up to 4 in the second circle. No A. swirskii were found in the third circle. Most $A$. swirskii stayed on the release plant. The number of $A$. swirskii on the release plant decreased over time $\left(F_{3,24}=15.01 ; P<0.0001\right)$. There was no difference between treatments $\left(F_{1,24}=1.35 ; \quad P=0.2570\right)$ and no interaction $\left(F_{3,24}=0.08 ; P=0.9701\right)$. This lack of differences may be explained by the finding that the plants without prey still had some thrips larvae ( $46 \pm 13$ ) on them, although significantly less than the plants with prey $(219 \pm 38)\left(F_{1,24}=29.91, P<0.0001\right)$. Thrips populations on the release plants were not reduced significantly over time $\left(F_{3,24}=1.42, P=0.2605\right)$.

The mean distance traversed from the release point was small ( $\max 8 \pm 3 \mathrm{~cm}$ after 14 days). There were no differences in mean distance traversed between treatments with and without prey $\left(F_{1,24}=1.23, P=0.2785\right)$. The distance traversed increased significantly over time $\left(F_{3,24}=8.60, P=0.0005\right)$. The interaction between days and treatment was not significant $\left(F_{3,24}=0.31, P=0.8159\right)$.

\subsection{Dispersal routes with and without prey}

Most A. swirskii were found on the plant or on the sticky card (Fig. 2). Almost none were recovered from the soil or the pot (although the sampling method could not extract dead mites from the soil). On plants without prey ( $0 \pm 0$ thrips larvae par plant), significantly less $A$. swirskii were found on the foliage as compared to the treatment with prey $(254 \pm 43$ thrips larvae par plant) $\left(F_{1,16}=22.40, P=0.0002\right)$. Also, numbers of $A$. swirskii recovered from the foliage declined over time in both treatments, leading
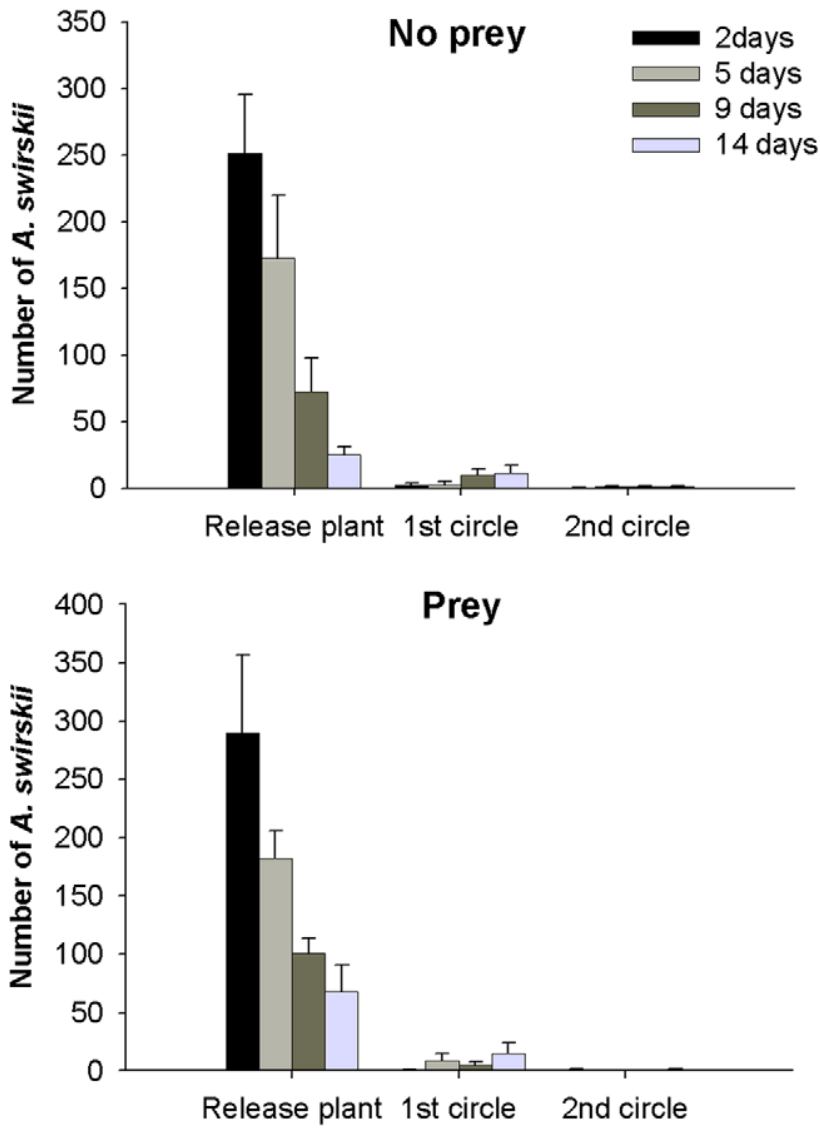

Fig. 1. Mean (+SE) number of Amblyseius swirskii in a patch of non-flowering potted chrysanthemums either with or without prey (Frankliniella occidentalis) measured at 2, 5, 9 and 14 days following predator release. The distance between the release plant and the first circle was $30 \mathrm{~cm}$, and the second circle was $60 \mathrm{~cm}$ (measured from the centre of the pots).

to significant differences among days $\left(F_{3,16}=24.79, P<0.0001\right)$. The interaction was not significant $\left(F_{3,16}=0.47, P=0.7082\right)$. On sticky cards, the number of $A$. swirskii that walked down or dropped off the plant and were captured on sticky cards increased significantly over time $\left(F_{3,16}=46.12, P<0.0001\right)$, but there were no significant differences between treatments with and without prey $\left(F_{1,16}=3.05, P=0.0997\right)$. The interaction was not significant $\left(F_{3,16}=1.82, P=0.1844\right)$. The $A$. swirskii were found scattered on the sticky cards under the plants, indicating that they dropped down rather than walked to the base of the pot.

\subsection{Effect of inter-plant contact on dispersal}

Plant to plant dispersal was greatly improved (2.5-7.8 times) when plants were touching (Fig. 3). A significantly higher percentage of the released $A$. swirskii was found to have reached the clean plant in the touching treatment $\left(F_{1,32}=66.40, P<0.0001\right)$. There were no differences among days $\left(F_{3,32}=1.61, P=0.2070\right)$. These results demonstrate that the mites distributed themselves quickly over the two plants when they were touching (within 2 days). The interaction was not significant $\left(F_{3,32}=1.69, P=0.1879\right)$.

\section{Discussion}

This study demonstrated that A. swirskii predatory mites did not disperse very far from the release site in a greenhouse crop of potted chrysanthemum. In the literature, dispersal distances of phytoseiid predatory mites vary greatly. Specialist species such as $P$. 

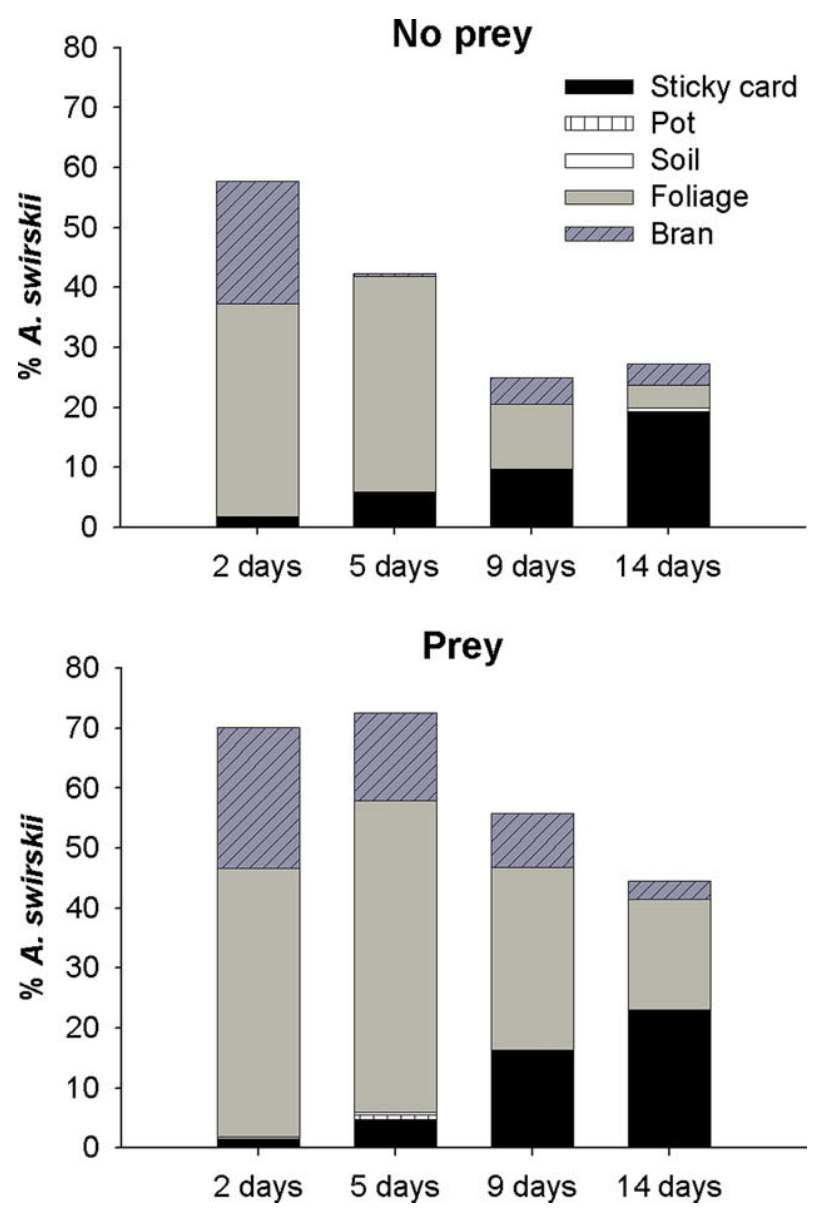

Fig. 2. Percentage of released Amblyseius swirskii recaptured on different parts of a single non-flowering potted chrysanthemum plant, either with or without prey (Frankliniella occidentalis), measured at 2, 5, 9 and 14 days following predator release.

persimilis seem to have more walking activity and aerial dispersal than generalist species (Jung and Croft, 2001). For example, van de Vrie (1985) reported that $P$. persimilis could disperse $10-15 \mathrm{~m}$ in 1 week in a chrysanthemum greenhouse both if the canopy was closed and across open spaces between beds. However, mean travel distances of $A$. swirskii in our dispersal study were similar to generalist predatory mites tested by Jung and Croft (2001) in a patch of bean plants (less than $10 \mathrm{~cm}$ after 5 days). Normal walking speed of $A$. swirskii on chrysanthemum leaves is $0.4 \mathrm{~mm} / \mathrm{s}$ (Buitenhuis, pers. obs.). In theory, $A$. swirskii would be able to walk $14 \mathrm{~cm}$ in one hour in a straight line. This indicates that other factors must have been limiting the dispersal distance of $A$. swirskii in the dispersal experiment.

One of the principal factors improving dispersal is increased inter-plant contact. Research has demonstrated that the greater the number of connections between adjacent plants, the greater the number of mites moving (Casey and Parrella, 2005; Skirvin and Fenlon, 2003; Zemek and Nachman, 1998, 1999). The dispersal route experiment in this study showed that $A$. swirskii tended to stay on the foliage of the plants and only a proportion of them eventually attempted to disperse by going down to the ground. The experiment testing the influence of inter-plant contact showed that when plants were touching, A. swirskii quickly dispersed over the two plants. When plants were not touching, only small numbers of $A$. swirskii were found on the clean plant. Predatory mites moving in the plant canopy usually have a lower risk of mortality, while movement from one isolated plant to another has an increased risk of mortality (Croft and Jung, 2001). When phytoseiid
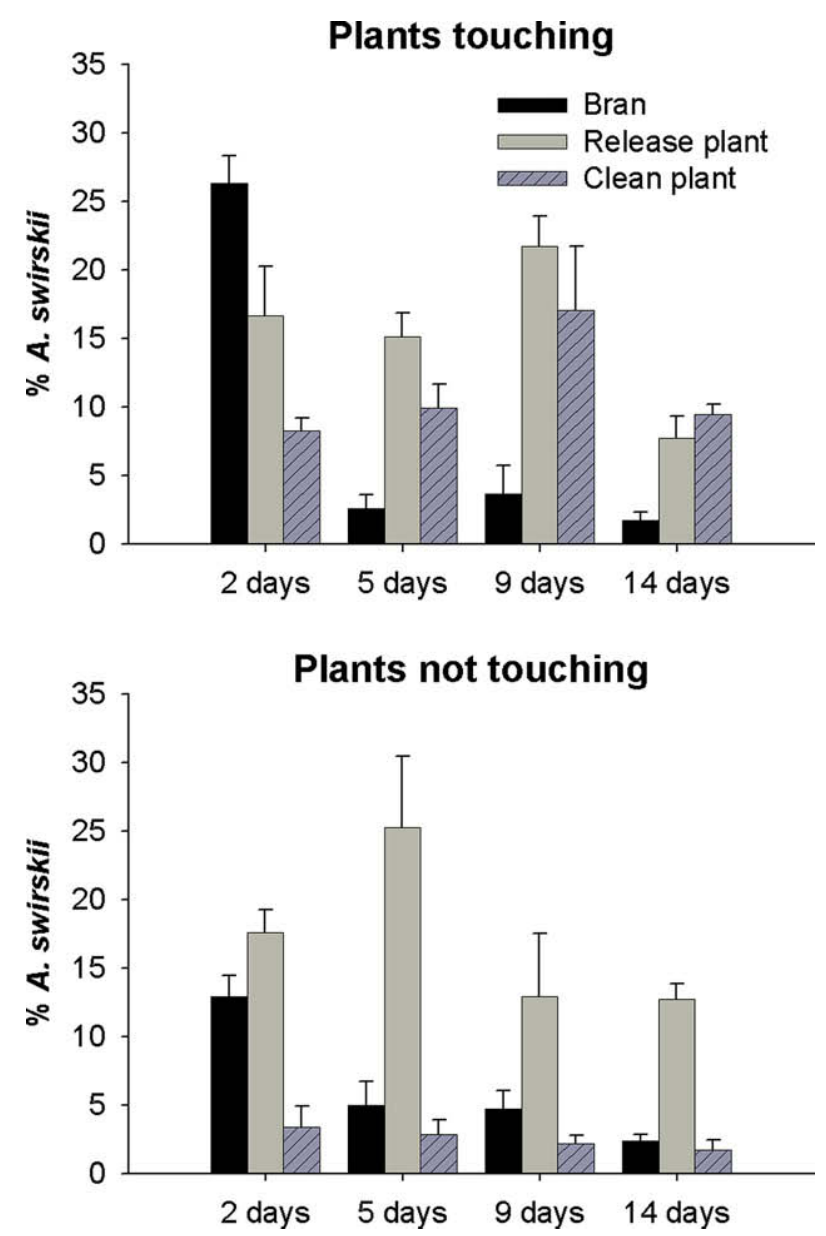

Fig. 3. Percentage (mean $+\mathrm{SE}$ ) of released Amblyseius swirskii recaptured on the release plant and the adjacent clean plant, where the plants were either touching or not, measured at 2, 5, 9 and 14 days following predator release.

mites had to move over a substrate, mortality occurred (Jung and Croft, 2000) and more mites were lost moving over a substrate than when plants were touching (Skirvin and Fenlon, 2003). In a release-recapture experiment where $A$. swirskii had to traverse $40 \mathrm{~cm}$ of soil substrate in order to reach plants, Nomikou et al. (2005) found that only a quarter of the released mites were recaptured on the plants and that it took the mites several days to get to the plants. In this study, testing the influence of inter-plant contact, only two plants were used. Additional experiments should measure the distance that $A$. swirskii would travel when all plants are connected.

The second factor affecting dispersal that was tested in this study was the presence or absence of prey. Some predator species such as $P$. persimilis, disperse less if prey is present (Zemek and Nachman, 1998, 1999) and dispersal is inversely related to prey density (Sabelis and Dicke, 1985). However, in trials in a greenhouse chrysanthemum crop, prey density did not seem to influence the dispersal distance of this predator species (Van de Vrie, 1985). Dispersal of Neoseiulus californicus (McGregor) (Acari: Phytoseiidae) was also reduced by the presence of prey (Auger et al., 1999). In the present study, the presence or absence of prey did not influence dispersal of $A$. swirskii. Although there was some contamination with thrips in the no-prey plots in the first experiment, the second experiment (dispersal routes) clearly pointed out that the presence of prey did not affect the number of $A$. swirskii found on sticky cards, indicating that there were no differences in the number of mites trying to disperse via the ground in the prey 
and no-prey treatments. The experiments did not test a situation where plants are touching with and without prey present, so it is possible that in this situation an influence of prey availability on dispersal might be found for A. swirskii. It is not known if $A$. swirskii is attracted to plants with thrips. However, research on whitefly prey indicates that $A$. swirskii searching behaviour is probably mostly random, with predators being arrested in patches with prey. Nomikou et al. (2005) showed that under certain circumstances (experience with leaves with whitefly prey, not starved) A. swirskii were attracted to volatiles of plants with whiteflies but this learned response waned after a few hours. For inexperienced A. swirskii, aggregation on plants with prey was probably due to differential arrestment of predators on plants with prey and on clean plants (Nomikou et al., 2005).

The presence of prey did improve survival of the predators on the plants in the dispersal route experiment. In the absence of food, A. swirskii will survive only 1 week (Buitenhuis, pers. obs.). There was no pollen or alternative prey, such as whiteflies or spider mites, on the plants in the experiments. However, A. swirskii is able to cannibalize conspecific mites to overcome situations where their natural prey is scarce (Rasmy et al., 2004), which might explain their continued presence on the plants without prey after 14 days.

Regarding the outcome of the experiments conducted in this study, it is clear that low dispersal rates of $A$. swirskii in potted ornamental crops increase the risks of pest outbreaks. Despite the fact that adult thrips are relatively weak fliers (Lewis, 1997), research has shown that the average spread of consecutive thrips generations in a greenhouse fluctuated between 0.05 and $0.17 \mathrm{~m}$ per day (Rhainds and Shipp, 2004). This means that the pest disperses faster than the predator. Therefore, it is important to ensure a good coverage of the crop at every predator introduction, especially when the foliage of the plants is not touching. Recent developments in release strategies of predatory mites aim to solve this problem. Several air blast systems have been developed (e.g., Koppert, 2009; Opit et al., 2005), growers experiment with sprinkler machines attached to the sprayer boom that rotate the bottles of predatory mites while they move over the crop (e.g., Biobest, 2008) and slow-release sachets of predatory mites have been produced in ribbons that can be drawn over the crop and act as bridges (e.g., Syngenta, 2008). In addition, establishing predatory mite populations on plants before the pots are moved out of the propagation greenhouse or using alternative food sources, such as pollen, should also improve pest suppression.

\section{Acknowledgments}

Thanks to Sam Fooks, Wonhyo Lee and Yun Zhang for technical assistance. Funding was obtained through an Agriculture Adaptation Council CORD IV Grant to Flowers Canada (Ontario) and Agriculture and Agri-Food Canada Matching Investment Initiative.

\section{References}

Auger, P. et al., 1999. Factors affecting ambulatory dispersal in the predaceous mite Neoseiulus californicus (Acari: Phytoseiidae). Experimental and Applied Acarology 23, 235-250.

Biobest, 2008. Biobolo. Bio Bulletin. Fall, 1.

Casey, C.A., Parrella, M.P., 2005. Evaluation of a mechanical dispenser and interplant bridges on the dispersal and efficacy of the predator, Phytoseiulus persimilis (Acari: Phytoseiidae) in greenhouse cut roses. Biological Control 32, 130-136.

Croft, B.A., Jung, C., 2001. Phytoseiid dispersal at plant to regional levels: a review with emphasis on management of Neoseiulus fallacis in diverse agroecosystems. Experimental and Applied Acarology 25, 763-784.

Croft, B.A., Kim, S.S., Kim, D.I., 1995. Leaf residency and interleaf movement of four Phytoseiid mites (Acari: Phytoseiidae) on apple. Environmental Entomology 24, 1344-1351.

Jung, C., Croft, B.A., 2000. Survival and plant-prey finding by Neoseiulus fallacis (Acari: Phytoseiidae) on soil substrates after aerial dispersal. Experimental and Applied Acarology 24, 579-596.

Jung, C., Croft, B.A., 2001. Ambulatory and aerial dispersal among specialist and generalist predatory mites (Acari: Phytoseiidae). Environmental Entomology 30, 1112-1118.

Koppert, 2009. Airbug. Available from: http://www.koppert.com/ Products_support.AIRBUG.14729+M5c99b1b7dc2.0.html.

Lewis, T., 1997. Flight and dispersal. In: Lewis, T. (Ed.), Thrips as Crop Pests. CAB International, New York, pp. 175-196.

McMurtry, J.A., Croft, B.A., 1997. Life-styles of phytoseiid mites and their roles in biological control. Annual Review of Entomology 42, 291-321.

Messelink, G.J., Van Steenpaal, S.E.F., Ramakers, P.M.J., 2006. Evaluation of phytoseiid predators for control of western flower thrips on greenhouse cucumber. Biocontrol 51, 753-768.

Nomikou, M., Janssen, A., Schraag, R., Sabelis, M.W., 2001. Phytoseiid predators as potential biological control agents for Bemisia tabaci. Experimental and Applied Acarology 25, 271-291.

Nomikou, M., Meng, R., Schraag, R., Sabelis, M.W., Janssen, A., 2005. How predatory mites find plants with whitefly prey. Experimental and Applied Acarology 36, 263-275.

Opit, G.P., Nechols, J.R., Margolies, D.C., Williams, K.A., 2005. Survival, horizontal distribution, and economics of releasing predatory mites (Acari: Phytoseiidae) using mechanical blowers. Biological Control: Theory and Application in Pest Management 33, 344-351.

Rasmy, A.H., Abou-El-Ella, G.M., Hussein, H.E., 2004. Cannibalism and interspecific predation of the phytoseiid mite, Amblyseius swirskii. Journal of Pest Science 77, 23-25.

Rhainds, M., Shipp, J.L., 2004. Dispersal by adult western flower thrips (Thysanoptera: Thripidae) in greenhouse crops. The Canadian Entomologist 136, 241-254.

Sabelis, M.W., Dicke, M., 1985. Long-range dispersal and searching behaviour. In: Helle, W., Sabelis, M.W. (Eds.), Spider Mites. Their Biology, Natural Enemies and Control. Elsevier, Amsterdam, pp. 141-160.

Skirvin, D., Fenlon, J., 2003. Of mites and movement: the effects of plant connectedness and temperature on movement of Phytoseiulus persimilis. Biological Control 27, 242-250.

Syngenta, 2008. Bugline swirskii. Control of whitefly and thrips. Available from: http://www.syngenta-bioline.co.uk/productdocs/html/BuglineS.htm.

Van Driesche, R.G., Heinz, K.M., 2004. An overview of biological control in protected culture. In: Heinz, K.M., Van Driesche, R.G., Parrella, M.P. (Eds.), Biocontrol in Protected Culture. Ball Publishing, Batavia, IL, pp. 1-24.

Van de Vrie, M., 1985. Greenhouse ornamentals. In: Helle, W., Sabelis, M.W. (Eds.), Spider Mites - Their Biology, Natural Enemies and Control, vol. 1B. Elsevier, Amsterdam, pp. 273-283.

Zemek, R., Nachman, G., 1998. Interactions in a tritrophic acarine predator-prey metapopulation system: effects of Tetranychus urticae on the dispersal rates of Phytoseiulus persimilis (Acarina: Tetranychidae, Phytoseiidae). Experimental and Applied Acarology 22, 259-278.

Zemek, R., Nachman, G., 1999. Interactions in a tritrophic acarine predator-prey metapopulation system: prey location and distance moved by Phytoseiulus persimilis (Acari: Phytoseiidae). Experimental and Applied Acarology 23, 21-40. 